\title{
A "kiss" before conception: triggering ovulation with kisspeptin-54 may improve IVF
}

\author{
Steven L. Young, MD, PhD \\ Division of Reproductive Endocrinology and Infertility, University of North Carolina, Chapel Hill, North Carolina, USA.
}

\begin{abstract}
A 30-year-old primigravid (C1P000) female with infertility secondary to her partner's oligospermia and her chronic anovulation presented 13 days after an oocyte retrieval for in vitro fertilization (IVF) with a positive home pregnancy test, abdominal distention, a 5-pound weight gain, nausea, shortness of breath, and reduced urinary frequency. Her IVF cycle included the usual cocktail for gonadotropin stimulation and was uncomplicated, except for excessively stimulated ovaries that led to a peak estradiol level of $6,000 \mathrm{pg} / \mathrm{ml}$ and the retrieval of $\mathbf{3 0}$ oocytes. Her past history was relevant only for anovulation due to polycystic ovarian syndrome (PCOS), though her preprocedure body mass index was normal at $21 \mathrm{~kg} / \mathrm{m}^{2}$. Pelvic ultrasound revealed abundant ascites and enlarged ovaries, at $8 \mathrm{~cm}$ average diameter. Serum human chorionic gonadotropin (hCG) concentration was 200 mIU/ml; she was hemoconcentrated (hemoglobin $16 \mathrm{~g} / \mathrm{dl}$ ), with normal liver function and coagulation testing. An ultrasound guided, transvaginal paracentesis removed 4 liters of straw-colored fluid, resulting in significant short-term symptom relief.
\end{abstract}

an exaggerated response to iatrogenic gonadotropin therapy (except in very rare cases). The development of moderate to severe symptoms is completely dependent on ovarian enlargement in addition to the elaboration of factors, such as VEGF, by the corpora lutea that cause capillary leakage. Stimulation of the corpora lutea by high levels of endogenous or exogenous hCG or luteinizing hormone ( $\mathrm{LH})$ exacerbates the problem by increasing the secretion of corpora lutea factors and maintaining ovarian size.

Because the development of severe OHSS is absolutely dependent on $\mathrm{LH}$ receptor stimulation by endogenous or exogenous LH or hCG, many strategies have been employed to limit the magnitude and duration of stimulation. Some strategies, such as the use of progesterone instead of hCG for luteal support or reducing the gonadotropin dose for patients with a high risk of OHSS, have been widely accepted because they do not adversely affect pregnancy rates (4). Other strategies, including the use of gonadotropinreleasing hormone $(\mathrm{GnRH})$ agonists to trigger endogenous $\mathrm{LH}$, are associated with a lower pregnancy rate and, thus, have not been as readily adopted (5). Although in vitro maturation of oocytes holds the promise to eliminate or limit the need for ovarian stimulation altogether, technical hurdles and relatively low efficacy of current oocyte maturation technologies greatly limit its use.

\section{Research advance}

In this issue, Jayasena and Abbara et al. present a new technique for triggering oocyte maturation in IVF that has potential to further reduce the risk of OHSS (6). These authors used a kisspeptin, kisspeptin-54, to stimulate an LH surge, which resulted in subsequent egg maturation, fertilization, production of a viable embryo, and pregnancy in a small cohort of women. Kisspeptins are a group of 
peptides produced through differential proteolytic processing of the KISS1 gene product. The kisspeptins and their G protein-coupled receptor, GPR54, stimulate GnRH secretion by hypothalamic neurons and play a critical physiological role in generating the LH surge (7). Therefore, functional absence of either receptor or ligand results in hypogonadotropic hypogonadism, while activating mutations of the receptor can cause precocious puberty (8). Furthermore, kisspeptin treatment of women with hypogonadotropic hypogonadism can result in resumption of gonadotropin secretion (9).

The report by Jayasena and Abbara and colleagues builds on previous data and suggests that kisspeptin can be used to generate an endogenous LH surge in assisted reproduction. Jayasena et al. designed a pilot dose-finding study that provides preliminary pharmacokinetics. Importantly, these preclinical, human data suggest that kisspeptin-54 administration results in favorable serum LH surge kinetics, efficient oocyte maturation, and competence to generate viable human embryos and babies (6). Further testing will be needed to determine whether the magnitude and duration of the LH surge generated by kisspeptin- 54 will be consistently optimal for assisted reproduction and reducing the risk of OHSS.

\section{Conclusions}

The use of kisspeptin and other neuropeptides and their analogues has the potential to replace or augment gonadotropins and improve efficacy, safety, and/ or convenience of ovarian stimulation
(10). This report by Jayasena and Abbara et al. is likely in the vanguard of a wave of new therapeutic options based on an increased understanding of the neuropeptide control of hypothalamic GnRH secretion. Interestingly, emerging data also demonstrate that these same neuropeptides have direct effects on the ovary and endometrium (11, 12), suggesting additional applications for these neuropeptides and their analogues. Over the last 50 years, the field of assisted reproduction has benefitted from continued innovation, rapidly applying scientific advances from the bench to the bedside, resulting in steady, significant improvement in the care of the infertile couple as well as those with other reproductive endocrine disorders. I expect that application of newer neuropeptide therapies will soon provide yet another significant advance in the treatment of reproductive disorders.

\section{Acknowledgments}

This work is supported by grant R01 HD067721 from the NIH and the Eunice Kennedy Shriver National Institute for Child Health and Human Development.

Address correspondence to: Steven Young, Division of Reproductive Endocrinology and Infertility, University of North Carolina, Chapel Hill, North Carolina 275997570, USA. Phone: 919.966.5283; E-mail: steven_young@med.unc.edu.

1. Mansour R, et al. International Committee for Monitoring Assisted Reproductive Technologies world report: Assisted Reproductive Technology
2006. Hum Reprod. 2014;29(7):1536-1551.

2. Practice Committee of American Society for Reproductive Medicine. Ovarian hyperstimulation syndrome. Fertil Steril. 2008; 90(5 suppl):S188-S193.

3. Assisted Reproductive Technology (ART). ART 2011 National Summary Report. CDC Web site. http://www.cdc.gov/art/ART2011. Updated: December 1, 2013. Accessed: June 25, 2014.

4. van der Linden M, Buckingham K, Farquhar C, Kremer JA, Metwally M. Luteal phase support for assisted reproduction cycles. Cochrane Database Syst. 2011;(10):CD009154.

5. Youssef MA, et al. Gonadotropin-releasing hormone agonist versus HCG for oocyte triggering in antagonist assisted reproductive technology cycles. Cochrane Database Syst. 2011;(1):CD008046.

6. Jayasena $\mathrm{CN}$, et al. Kisspeptin-54 triggers egg maturation in women undergoing in vitro fertilization. J Clin Invest. 2014;124(8):3667-3677.

7. Skorupskaite K, George JT, Anderson RA. The kisspeptin-GnRH pathway in human reproductive health and disease. Hum Reprod Update. 2014;20(4):485-500.

8. Silveira LG, Latronico AC, Seminara SB. Kisspeptin and clinical disorders. Adv Exp Med Biol. 2013;784:187-199.

9. Jayasena $\mathrm{CN}$, et al. Twice-weekly administration of kisspeptin-54 for 8 weeks stimulates release of reproductive hormones in women with hypothalamic amenorrhea. Clin Pharmacol Ther. 2010;88(6):840-847.

10. Millar RP, Newton CL. Current and future applications of $\mathrm{GnRH}$, kisspeptin and neurokinin B analogues. Nat Rev Endocrinol. 2013; 9(8):451-466.

11. Gaytan F, et al. Kisspeptin receptor haplo-insufficiency causes premature ovarian failure in spite of preserved gonadotropin secretion [published online ahead of print June 2, 2014]. Endocrinology. doi:10.1210/en.2014-1110.

12. Cejudo Roman A, et al. Analysis of the expression of neurokinin B, kisspeptin, and their cognate receptors NK3R and KISS1R in the human female genital tract. Fertil Steril. 2012; 97(5):1213-1219. 